\title{
Classical and $a b$ initio preparation of reliable structures for polymeric coordination compounds
}

\author{
Harald O. Jeschke * L. Andrea Salguero, and Roser Valentí \\ Institut für Theoretische Physik, Johann Wolfgang Goethe-Universität, \\ Max-von-Laue-Str. 1, 60438 Frankfurt/Main, Germany \\ Christian Buchsbaum, Martin U. Schmidt, and Matthias Wagner \\ Institut für Anorganische und Analytische Chemie, Johann Wolfgang Goethe-Universität, \\ Max-von-Laue-Str. 7, 60438 Frankfurt/Main, Germany
}

(Dated: June 22, 2017)

\begin{abstract}
The detailed investigation of electronic and magnetic properties of polymeric coordination materials with accurate $a b$ initio quantum mechanical methods is often computationally extremely demanding because of the large number of atoms in the unit cell. Moreover, usually the available structural data are insufficient or poorly determined especially when the structure contains hydrogen atoms. In order to be able to perform controlled $a b$ initio calculations on reliable structures, we use a two step approach to systematically prepare model structures for polymeric coordination compound systems and to relax them to their equilibrium configuration. First, a structure is constructed on the basis of a crystallographic database and optimized by force field methods; in the second step, the structure is relaxed by ab initio quantum mechanical molecular dynamics. With this structure we perform accurate electronic structure calculations. We will apply this procedure to a $\mathrm{Fe}(\mathrm{II})$ triazole compound and to a coordination polymer of $\mathrm{Cu}(\mathrm{II})$ ions with 2,5-bis(pyrazol-1yl)-1,4-dihydroxybenzene.
\end{abstract}

PACS numbers: $61.66 . \mathrm{Hq}, 71.20 . \mathrm{Rv}, 75.30 . \mathrm{Et}, 75.50 . \mathrm{Xx}$

Keywords: Coordination polymer, density functional theory, structure optimization

\section{INTRODUCTION}

Polymeric complexes form a class of materials that attract interest from chemists and physicists. The possibility to apply organic synthesis methods to produce families of compounds with similar structures but subtly differing physical behavior offers an opportunity to design materials with planned properties.

Materials showing temperature or pressure induced high-spin - low-spin transitions are currently being discussed for applications in data storage or spintronics. Such magnetic transitions can be optically triggered, raising hopes for technological applications. The cooperativity of such light-induced phase transitions also raises questions concerning the underlying microscopic processes $1,2,3,4$. Another important class of polymeric coordination compounds are, in general, low-dimensional magnetic materials where the coupling strengths between the metal ions can be tuned by chemically modifying the ligands and the transition metal ion coordination. Effects of, for instance, dimensional crossover can be studied in this way.

Most of these compounds generally contain a large number of atoms in the unit cell and a very low symmetry (in $80 \%$ of the cases triclinic or monoclinic) making the first principles study of the electronic and magnetic properties of these systems under different conditions (pressure, temperature, light irradiation) computationally very demanding. Moreover, since an accurate determination of hydrogen positions with X-ray diffractometry is very difficult, usually the available structure data are poorly determined implying unstable quantum mechanical calculations. For some cases, the crystal structures are not even known, due to absence of single crystals suitable for an X-ray structure analysis. In order to overcome these shortcomings, we use in this communication a two-step approach which allows to perform accurate $a b$ initio density functional theory (DFT) calculations on reliable model structures for coordination polymers. It should be noted, that the expression " $a b$ initio prediction of crystal structures" is also used in the sense of "a priori prediction", i.e. predictions without reference to diffraction data. Possible crystal structures of organic compounds with 20-60 atoms can be predicted within some days or weeks using force field methods 5 . This works also for organometallic compounds, e.g. pentamethylferrocene $\mathrm{Fe}\left(\mathrm{C}_{5} \mathrm{H}_{5}\right)\left[\left(\mathrm{C}_{5} \mathrm{CH}_{3}\right)_{5}{ }^{16}\right.$. Quantum mechanical methods are much more time-consuming; hence their use is limited to local optimizations starting from a given structural model. Thus, quantum mechanical and force field methods can be combined by making first a global optimization using force field methods, followed by a local optimization with quantum mechanical methods. This was done for many inorganic systems, e.g. $\mathrm{NaCl}$ or $\mathrm{MgF}_{2}{ }^{7}$, and a few simple organic compounds, e.g. glycole $\mathrm{C}_{2} \mathrm{H}_{4}(\mathrm{OH})_{2}$ and glycerol $\mathrm{C}_{3} \mathrm{H}_{5}(\mathrm{OH})_{3}{ }^{8}$. In most other cases of crystal structure predictions, quantum mechanical calculations were only used for secondary calculations, e.g. to calculate molecular geometries and electrostatic charges, or to derive intramolecular or intermolecular potential curves for subsequent force field optimizations 9 . 
If experimental data are known, e.g. from crystal data of analogous structures, this knowledge should of course be used as far as possible in order to generate reliable model structures and to calculate solid state properties. Here, we apply $a b$ initio DFT methods to understand the relationships between the structures and the electronic and magnetic properties of both i) spin crossover materials and ii) low-dimensional spin systems. We will consider two representatives from this large class of compounds: i) a Fe(II)triazole compound ${ }^{10,11}$ which is thought to have a polymer structure and ii) a coordination polymer of $\mathrm{Cu}(\mathrm{II})$ ions with 2,5bis(pyrazol-1-yl)-1,4-dihydroxybenzene ${ }^{12}$, (we shall denote it as $[\mathrm{Cu}(\text { bpydhb })]_{n}$, alternative names given in the literature are $\left.\mathrm{CuCCP}^{13}\right)$.

For the Fe(II)triazole system ${ }^{10,11}$, there is no direct accurate determination of the crystal structure of the complex as it cannot be crystallized sufficiently well. A chain-like structure for such compounds of formula $\left[\mathrm{Fe}(\mathrm{Rtrz})_{3}\right] \mathrm{A}_{2} . \mathrm{S}$ where A denotes the anion was predicted from EXAFS spectroscopy ${ }^{14}$. For analogous $\mathrm{Cu}(\mathrm{II})$ compounds this chain structure was confirmed by single crystal X-ray analysis (see e.g. Refs. 15,16). Polymeric iron triazole compounds always show a poor crystallinity, and single crystals cannot be grown. Hence the X-ray structure analyses are limited to molecular compounds, e.g. trimers containing three Fe(II) ions connected by 6 triazole ligands and 6 terminal water molecules ${ }^{17}$. Polymeric iron triazole complexes have been studied by powder diffraction; but apart from a preliminary indexing of $\left[\mathrm{Fe}(\mathrm{Htrz})_{3}\right]\left(\mathrm{ClO}_{4}\right)_{2} \cdot 1.85 \mathrm{H}_{2} \mathrm{O}^{18}$, no structural data have been published so far. On the other hand, these polymeric compounds show interesting high-spin-lowspin transitions in the solid state (see e.g. Ref. 19), and quantum mechanical calculations could help to understand the mechanism of this spin crossover. Since all calculations of electronic and magnetic properties require a reliable and reasonable model of the crystal structure, we constructed the structures of a hypothetical polymer crystal. All available information of molecular iron triazole systems, and of analogous $\mathrm{Cu}(\mathrm{II})$ polymers was included. In order to make the $a b$ initio DFT calculations feasible we constructed at first some models containing small triazoles (e.g. methyl-triazole) and simple anions (e.g. $\mathrm{F}^{-}$). Our rationale is that the model compound still has the same coordination and immediate environment of the $\mathrm{Fe}$ (II) ion as the real material which should thus allow us to study the mechanisms of the high-spin - low-spin transition. Structures with more complex anions will be considered later.

The second system that we study, the coordination polymer of $\mathrm{Cu}(\mathrm{II})$ ions with 2,5-bis(pyrazol-1-yl)-1,4dihydroxybenzene, $[\mathrm{Cu}(\text { bpydhb })]_{n}$, has been recently synthesized by Wagner et al ${ }^{12}$ The $\mathrm{Cu}^{2+}$ ions in this compound form spin-1/2 antiferromagnetic Heisenberg chains running along the z-axis. We will analyze the influence on the electronic properties of slight structural modifications, like substitutions of small organic groups on the dihydroxybenzene or introduction of water ligands which change the coordination of $\mathrm{Cu}$ from square to octahedral. The structure of $\mathrm{Cu}(\mathrm{II})$ with 2,5-bis(pyrazol1-yl)-1,4-dihydroxybenzene has been obtained by X-ray powder diffraction ${ }^{12}$ and we will apply our two-step approach for the modified structures based on these data.

\section{METHODS}

We derive and investigate model polymeric coordination compound structures in two steps. First, we create a rough structure on the basis of crystallographic databases 21 . If no crystal structures of similar compounds are known - like in the case of Fe triazoles we construct a hypothetical crystal structure having the highest possible symmetry, a small number of atoms per unit cell and a sensible arrangement of the individual fragments. The crystal structure is optimized by force field methods including the optimization of the unit cell parameters, but maintaining the crystallographic symmetry. We used the Cerius ${ }^{2}$ program package ${ }^{22}$ with a modified Dreiding 23 force field; atomic charges were calculated by the Gasteiger method ${ }^{24}$. Afterwards we proceed to improve the structure by relaxing it again, but now with an $a b$ initio quantum mechanical molecular dynamics method 25 . Note that we employ an all electron method already at this step in order to obtain the best possible equilibrium structure even for demanding transition metal complexes. Finally, we analyze the properties of the relaxed model structure with an all electron $a b$ initio DFT method of the highest precision ${ }^{26}$.

Our Car Parrinello (CP) ab initio molecular dynamics (AIMD) calculations ${ }^{25}$ are performed with a projector augmented wave (PAW) basis set ${ }^{27}$. This is an all electron calculation based on the frozen core approximation. The exchange-correlation potential is evaluated in the generalized gradient approximation (GGA) as parametrized in Ref. 28. After a first analysis of the electronic properties for the classically prepared model in order to determine the structural stability, we employ an AIMD with friction to refine the structure. We impose constraints on the atomic coordinates in order to preserve the space group symmetry of the compound.

For the final analysis step we employ the full potential linearized augmented plane wave (LAPW, APW + lo) method as implemented in the WIEN2k code ${ }^{26}$.

\section{RESULTS}

\section{A. Fe(II)-Triazole}

The Fe(II) spin crossover molecular systems have been intensively studied in the past ${ }^{19}$ and various theories have been developed about the mechanism of the high-spin low-spin transition. A few years ago, Kahn et al. ${ }^{10}$ proposed that the cooperativity of this transition may be en- 
hanced by replacing molecular crystals by polymers, since in the latter the metallic sites are bridged by chemical linkers through which one may get efficient intersite coupling. A first principles DFT study of such systems would bring some light into the properties of these materials. Unfortunately, to date it has not been possible to get an accurate X-ray structure determination of Fe(II) spin crossover polymer systems, as discussed above, which is an indispensable input for $a b$ initio DFT calculations and therefore, to our knowledge, no reliable ab initio studies were performed. With the two-step method, we have obtained - based on known data for molecular Fe(II) spin crossover systems - optimized model polymer structures which can be used as input for $a b$ initio DFT calculations. In Fig. 1 we present the structure of the polymer Fe(II) compound with 4-methyl-1,2,4-triazole moieties as bridging ligands between the $\mathrm{Fe}(\mathrm{II})$ cations. As anions we chose simple $\mathrm{F}^{-}$ions in order not to complicate the DFT calculations. The structure has $P 2_{1} / m$ symmetry; a higher symmetry was not possible with this chemical composition. As an initial structure with low spin character, the distances between the Fe(II) centers and the neighboring $\mathrm{N}$ atoms of the triazole rings were chosen to be $d_{\mathrm{Fe}-\mathrm{N}}=1.97 \AA$, close to the Fe-N distance of $d_{\mathrm{Fe}-\mathrm{N}}=2.0 \AA$ that is known to be typical for the low spin variants of Fe triazole complexes ${ }^{20}$ while for the structure with high spin character we chose $d_{\mathrm{Fe}-\mathrm{N}}=2.2 \AA$. In Fig. 2 we present the spin-polarized density of states (DOS) obtained ${ }^{26}$ for this system after structural optimization for the low spin (Fig. 2 (a)) and the high spin (Fig. 2 (b)) states. Shown is the total Fe $d$ DOS for majority (upper panel) and minority (lower panel) spin states. Since Fe is in an octahedral environment of $\mathrm{N}$, the $3 d$ states split into lower $t_{2 g}\left(d_{x y}, d_{x z}, d_{y z}\right)$ and higher $e_{g}\left(d_{x^{2}-y^{2}}, d_{z^{2}}\right)$ states. In Fig. 2 (a) the 6 electrons of $\mathrm{Fe}(\mathrm{II}) 3 d^{6}$ occupy fully the $t_{2 g}$ states (see DOS manifold between -2 and $-0.2 \mathrm{eV}$ ) for both spins thus corresponding to a low spin $\mathrm{S}=0$ state. The $e_{g}$ states are completely empty and lie above the Fermi level. In Fig. 2 (b), the majority (spin up) $t_{2 g}$ and $e_{g}$ states are occupied (upper panel DOS in the range between $-3.8 \mathrm{eV}$ and $-0.6 \mathrm{eV}$ ) while the minority (spin down, lower panel) $t_{2 g}$ states are about one-third occupied and two-thirds empty. The minority $e_{g}$ states are completely empty (states in the range of energies between $1 \mathrm{eV}$ and $3 \mathrm{eV}$, lower panel). These results indicate that the system is in a high spin $\mathrm{S}=2$ state. A detailed investigation of the magnetic properties of this polymer system for both spin states will be presented elsewhere ${ }^{29}$.

\section{B. Cu(II)-Polymer}

The $\mathrm{Cu}(\mathrm{II})$ coordination polymer $\left[\mathrm{Cu}(\text { bpydhb) }]_{n}\right.$ synthesized by Wagner et al $\frac{12}{\underline{12}}$ crystallizes in the triclinic space group $P \overline{1}$ (No. 2) and consists of stacks of chains of deprotonated 2,5-bis(pyrazol-1-yl)-1,4-dihydroxybenzene molecules connected by $\mathrm{Cu}^{2+}$ ions. This system behaves as a homogeneous antiferromagnetic spin- $1 / 2$ Heisenberg

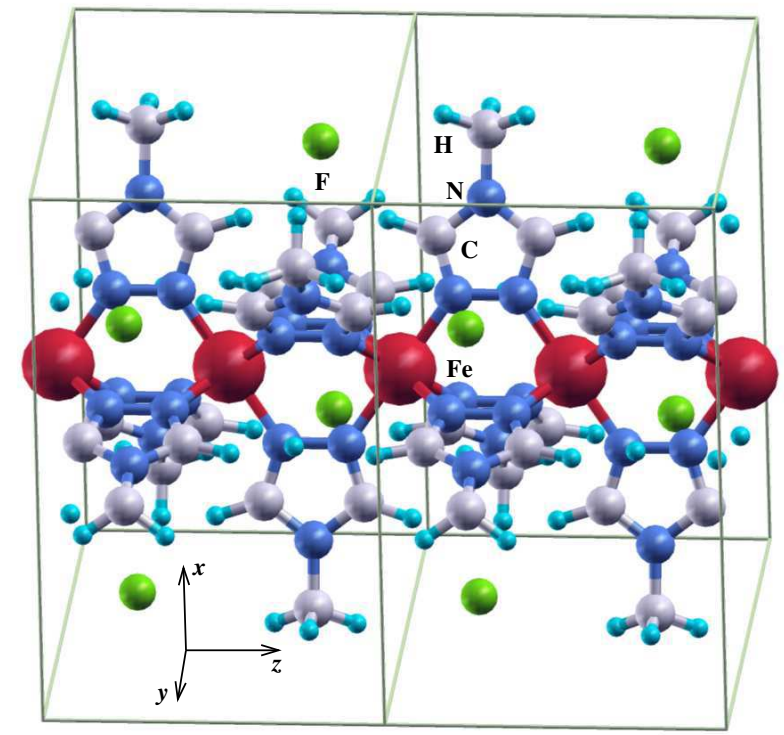

Figure 1: Two unit cells of the triazole model polymer. The usually complicated anions have been replaced by single atom $\mathrm{F}^{-}$anions.

chain with an exchange coupling constant $J=21.5 \mathrm{~K} / \mathrm{k}_{\mathrm{B}}$ estimated from magnetic susceptibility measurements 13 . The modular nature of this polymer makes it an adequate candidate to study chemically feasible structural modifications which may change the electronic and magnetic properties. Of special interest is the effect of dimensionality, i.e. tuning the crossover from one dimension to higher dimensions with the inclusion of adequately modified ligands. Since in this communication we want to stress the procedure we follow to design these structures and how we calculate the electronic properties, we will concentrate here on one substitution, namely two hydrogen atoms in the hydroquinone ring by two amino groups and show the efficiency of the two-step process. A second issue in this context that requires some attention is the study of the effect of two additional ligands at the $\mathrm{Cu}(\mathrm{II})$ ion, since such an octahedral coordination of copper is also frequently observed. We will here present the relaxation process on $[\mathrm{Cu}(\text { bpydhb })]_{n}$ with two additional water molecules in the environment of copper.

In Fig. 3 we present the relaxed structure of $[\mathrm{Cu}(\text { bpydhb })]_{n}$ where two hydrogen atoms in the hydroquinone rings have been substituted by two amino groups. This structure was obtained first by applying the force field method, which already has the correct information of the chemical distances and angles from data bases. A quantum mechanical evaluation of the forces on the atoms (see Table I) for this structure, performed with the full potential LAPW method 26 (FPLAPW) still shows an unstable structure. Our aim is to relax this structure showing forces in the hundreds of $\mathrm{mRyd} / \mathrm{a}_{\mathrm{B}}$ with quantum mechanical molecular dynamics calculations, AIMD, until we find forces smaller than $25 \mathrm{mRyd} / \mathrm{a}_{\mathrm{B}}$ which we set as upper bound for the force values. In order to get 

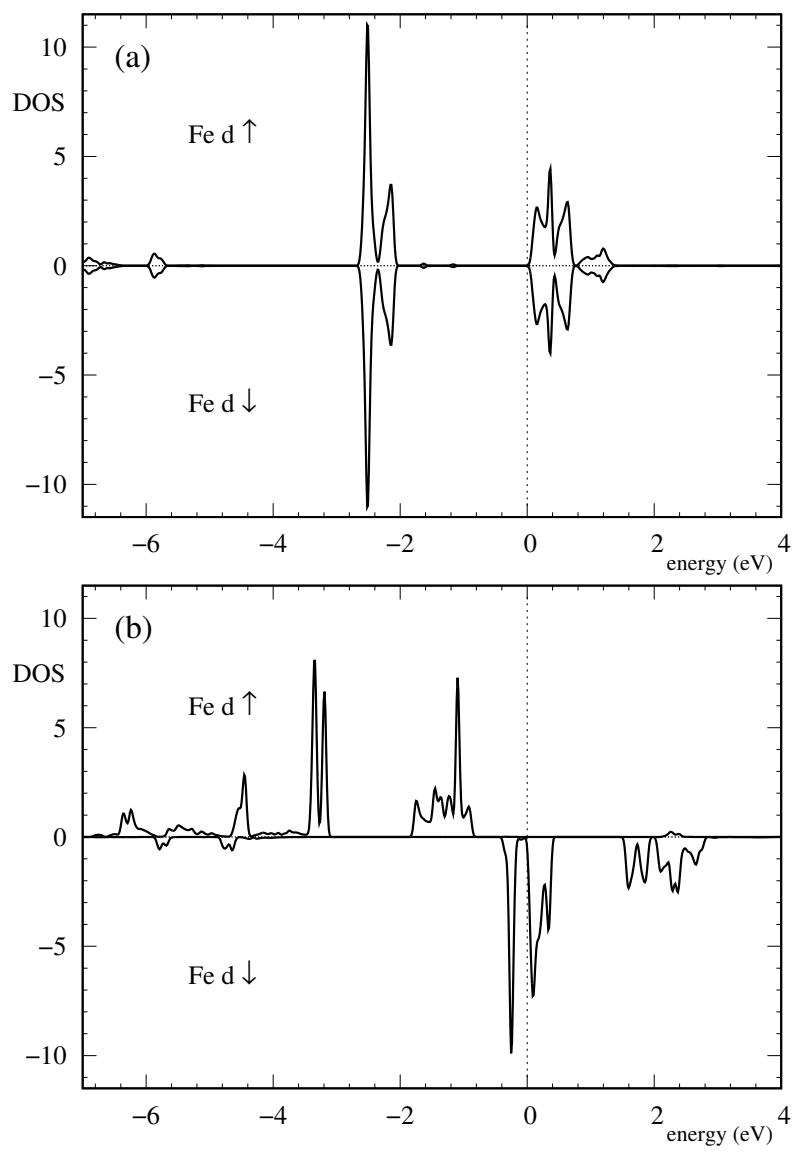

Figure 2: Spin polarized $\mathrm{Fe} d$ density of states of the $\mathrm{Fe}$ triazole structure Fig. 1 in units of states/electronvolt/atom/spin. (a) for the $S=0$ state of $\mathrm{Fe}$ (b) for the $\mathrm{S}=2$ state of Fe. The upper and lower panels of the figure show respectively the density of states for the majority and minority spin states. The Fermi energy is indicated by a dotted line.

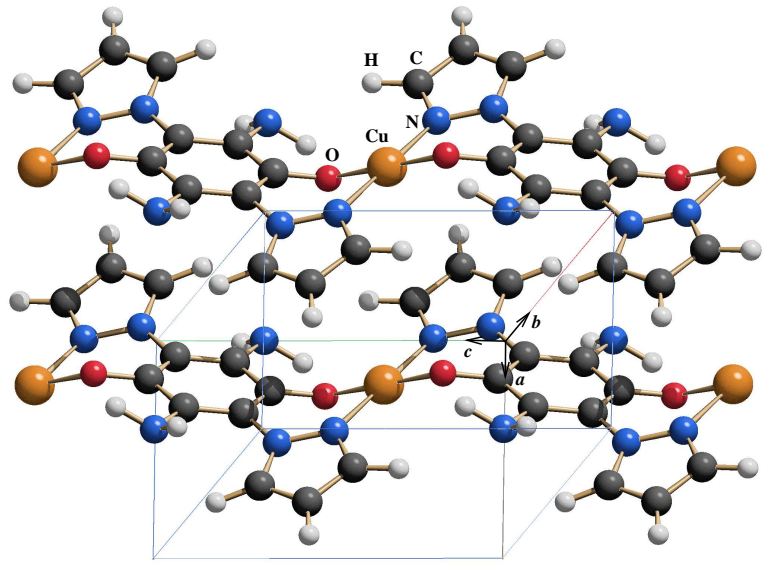

Figure 3: Four unit cells of $\left[\mathrm{Cu}(\text { bpydhb) }]_{n}\right.$ (see text for the detailed formula) where two hydrogen atoms in the hydroquinone rings have been substituted by two amino groups. We find that the relaxed structure shows some tilting of the amino groups out of the plane defined by the benzene ring.
Table I: Forces $^{26}$ between the atoms for the $\mathrm{Cu}(\mathrm{II})-\mathrm{NH}_{2}$ polymer structure designed with the force field method. Note that the forces were determined under the constraint of inversion symmetry; thus there are no forces acting on the $\mathrm{Cu}$ (II) ion center. Units are in $\mathrm{mRyd} / \mathrm{aB}$. See TableIII for identification of the atom location.

\begin{tabular}{crrr}
\hline Atom & $\mathrm{F}_{x}$ & $\mathrm{~F}_{y}$ & $\mathrm{~F}_{z}$ \\
\hline \hline $\mathrm{Cu}$ & 0 & 0 & 0 \\
$\mathrm{O}$ & -47.4 & -62.7 & 51.3 \\
$\mathrm{~N} 1$ & 89.1 & 32.8 & -42.6 \\
$\mathrm{~N} 2$ & 22.4 & 27.2 & -95.8 \\
$\mathrm{~N} 3$ & -94.2 & -66.8 & 33.5 \\
$\mathrm{C} 1$ & 35.0 & 22.9 & 1.8 \\
$\mathrm{C} 2$ & -92.7 & -29.2 & 111.1 \\
$\mathrm{C} 3$ & -39.2 & -41.7 & 294.8 \\
$\mathrm{C} 4$ & -239.7 & -104.1 & 16.5 \\
$\mathrm{C} 5$ & -26.6 & -12.8 & -304.2 \\
$\mathrm{C} 6$ & 106.7 & 88.9 & 46.0 \\
$\mathrm{H} 1$ & 128.6 & -28.7 & 215.0 \\
$\mathrm{H} 2$ & 255.3 & 106.1 & -34.2 \\
$\mathrm{H} 3$ & 23.8 & 25.6 & -279.0 \\
$\mathrm{H} 4$ & 4.8 & 10.8 & -18.3 \\
$\mathrm{H} 5$ & -16.2 & 64.5 & 56.3 \\
\hline
\end{tabular}

structures with forces smaller than $1 \mathrm{mRyd} / \mathrm{a}_{\mathrm{B}}$ within the FPLAPW method, a fine tuning between the various first principles DFT methods we employ has to be considered. For our purposes here, a benchmark of $25 \mathrm{mRyd} / \mathrm{a}_{\mathrm{B}}$ is reasonable.

In Table II the forces after the AIMD relaxation are shown and we observe that the structure is relaxed to our required precision. The fractional coordinates of this structure are given in Table III where the symmetry and cell parameters were kept unchanged with respect to the unsubstituted $\mathrm{Cu}$ polymer system $[\mathrm{Cu}(\text { bpydhb })]_{n}{ }^{12} a=$ $5.1723 \AA, b=7.9587 \AA, c=8.2298 \AA$, and angles $\alpha=$ $118.221^{\circ}, \beta=91.520^{\circ}, \gamma=100.148^{\circ}$.

In a different modification of the $\mathrm{Cu}(\mathrm{II})$ polymer structure, we want to investigate the influence of a change of the $\mathrm{Cu}$ coordination from four to six on the electronic properties of the $\mathrm{Cu}$ (II) polymer. We added two water molecules bound to the $\mathrm{Cu}$ center and proceeded as outlined for the case of the $\mathrm{NH}_{2}$ substitution: A first structure was obtained from the force field method, yielding high forces when we controlled it with an FPLAPW calculation. Further relaxation with AIMD yields forces around $20 \mathrm{mRyd} / \mathrm{a}_{\mathrm{B}}$. The corresponding structure is shown in Fig. 4, and the fractional coordinates are given in Table IV] The new unit cell is triclinic with space group $P \overline{1}$ (No. 2) as in the original $\mathrm{Cu}(\mathrm{II})$ polymer. The cell parameters are $a=5.2341 \AA, b=11.2493 \AA$, $c=8.0721 \AA$ and the angles are $\alpha=117.6108^{\circ}, \beta=$ $68.8218^{\circ}, \gamma=127.1551^{\circ}$. Note the variation of these parameters with respect to those of $[\mathrm{Cu}(\text { bpydhb })]_{n}{ }^{12}$, since in the relaxation process of the new structure the unit cell 
Table II: Forces $^{26}$ between the atoms for the relaxed $\mathrm{Cu}$ (II)$\mathrm{NH}_{2}$ polymer. Units are $\mathrm{mRyd} / \mathrm{aB}$.

\begin{tabular}{crrr}
\hline Atom & $\mathrm{F}_{x}$ & $\mathrm{~F}_{y}$ & $\mathrm{~F}_{z}$ \\
\hline \hline $\mathrm{Cu}$ & 0 & 0 & 0 \\
$\mathrm{O}$ & 12.7 & 16.9 & 21.7 \\
$\mathrm{~N} 1$ & 3.9 & 0.7 & -17.2 \\
$\mathrm{~N} 2$ & 12.5 & -2.3 & 8.4 \\
$\mathrm{~N} 3$ & -21.0 & -9.4 & -5.5 \\
$\mathrm{C} 1$ & -1.0 & -9.3 & -19.4 \\
$\mathrm{C} 2$ & 10.3 & 3.6 & -2.1 \\
$\mathrm{C} 3$ & -7.1 & -7.0 & 14.6 \\
$\mathrm{C} 4$ & -10.6 & -12.7 & -3.1 \\
$\mathrm{C} 5$ & -12.0 & -6.0 & -19.5 \\
$\mathrm{C} 6$ & 12.0 & 3.4 & -0.7 \\
$\mathrm{H} 1$ & 1.4 & -0.7 & 3.5 \\
$\mathrm{H} 2$ & 3.1 & -1.7 & -1.4 \\
$\mathrm{H} 3$ & -0.4 & -0.3 & -4.1 \\
$\mathrm{H} 4$ & 0.08 & -5.5 & -0.4 \\
$\mathrm{H} 5$ & 1.1 & -0.9 & 0.5 \\
\hline
\end{tabular}

Table III: Fractional atomic positions of nonequivalent atoms in $\mathrm{Cu}(\mathrm{II})-\mathrm{NH}_{2}$ obtained after relaxation. The corresponding unit cell parameters are given in the text.

\begin{tabular}{cccc}
\hline Atom & $x$ & $y$ & $z$ \\
\hline \hline $\mathrm{Cu}$ & 0.5 & 0.5 & 0.5 \\
$\mathrm{O}$ & 0.45952338 & 0.34384463 & 0.62047714 \\
$\mathrm{~N} 1$ & 0.87129861 & 0.67649196 & 0.85703667 \\
$\mathrm{~N} 2$ & 0.81592808 & 0.67403219 & 0.69273582 \\
$\mathrm{~N} 3$ & 0.89342131 & 0.81953377 & 0.25313235 \\
$\mathrm{C} 1$ & 0.48379900 & 0.42607948 & 0.80332348 \\
$\mathrm{C} 2$ & 0.68236558 & 0.58873536 & 0.92870320 \\
$\mathrm{C} 3$ & 0.03998649 & 0.76736537 & 0.66677421 \\
$\mathrm{C} 4$ & 0.23751744 & 0.83563448 & 0.81694695 \\
$\mathrm{C} 5$ & 0.12514376 & 0.77281287 & 0.93447831 \\
$\mathrm{C} 6$ & 0.70113966 & 0.66249861 & 0.12137891 \\
$\mathrm{H} 1$ & 0.20669437 & 0.78002257 & 0.06146726 \\
$\mathrm{H} 2$ & 0.43843580 & 0.91660682 & 0.83522646 \\
$\mathrm{H} 3$ & 0.04936408 & 0.77481595 & 0.53807198 \\
$\mathrm{H} 4$ & 0.82019516 & 0.86915420 & 0.37967013 \\
$\mathrm{H} 5$ & 0.94873662 & 0.93065156 & 0.22239484 \\
\hline
\end{tabular}

was allowed to relax properly in order to accommodate the water molecules.

We investigate now the electronic properties of the two model $\mathrm{Cu}(\mathrm{II})$ polymers with a precise FPLAPW calculation. A detailed account of the electronic and magnetic properties of both systems is presented elsewhere $e^{30}$. Here we would like to stress the effect of a planar $\mathrm{Cu}$ environment (for the $\mathrm{Cu}-\mathrm{NH}_{2}$ case) in comparison of an octahedral environment (for the $\mathrm{Cu}-\mathrm{H}_{2} \mathrm{O}$ case) on the $\mathrm{Cu} d$ density of states. In Figs. 5 (a) and (b) the $\mathrm{Cu} d$ density
Table IV: Fractional atomic positions of nonequivalent atoms in $\mathrm{Cu}$ (II)- $\mathrm{H}_{2} \mathrm{O}$ obtained after relaxation. The corresponding unit cell parameters are given in the text.

\begin{tabular}{cccc}
\hline Atom & $x$ & $y$ & $z$ \\
\hline \hline $\mathrm{Cu}$ & 0.5 & 0.5 & 0.5 \\
$\mathrm{O} 1$ & 0.54548630 & 0.39146013 & 0.61449576 \\
$\mathrm{O} 2$ & 0.11104809 & 0.50997119 & 0.71612732 \\
$\mathrm{C} 1$ & 0.50846703 & 0.44754970 & 0.80067207 \\
$\mathrm{C} 2$ & 0.68032852 & 0.61183880 & 0.90724635 \\
$\mathrm{C} 3$ & 0.05894527 & 0.84496851 & 0.61704992 \\
$\mathrm{C} 4$ & 0.26950758 & 0.95163087 & 0.75697623 \\
$\mathrm{C} 5$ & 0.15279255 & 0.87395244 & 0.88376263 \\
$\mathrm{C} 6$ & 0.32801067 & 0.33993413 & 0.89980571 \\
$\mathrm{~N} 1$ & 0.88662263 & 0.72926141 & 0.81944391 \\
$\mathrm{~N} 2$ & 0.82847386 & 0.71119262 & 0.65595860 \\
$\mathrm{H} 1$ & 0.23644016 & 0.90962503 & 0.01364916 \\
$\mathrm{H} 2$ & 0.47761970 & 0.07047826 & 0.76570604 \\
$\mathrm{H} 3$ & 0.05611367 & 0.85699006 & 0.49132676 \\
$\mathrm{H} 4$ & 0.20991897 & 0.21421176 & 0.81558567 \\
$\mathrm{H} 5$ & 0.02924495 & 0.44665601 & 0.79637256 \\
$\mathrm{H} 6$ & 0.92725690 & 0.45505960 & 0.64249638 \\
\hline
\end{tabular}

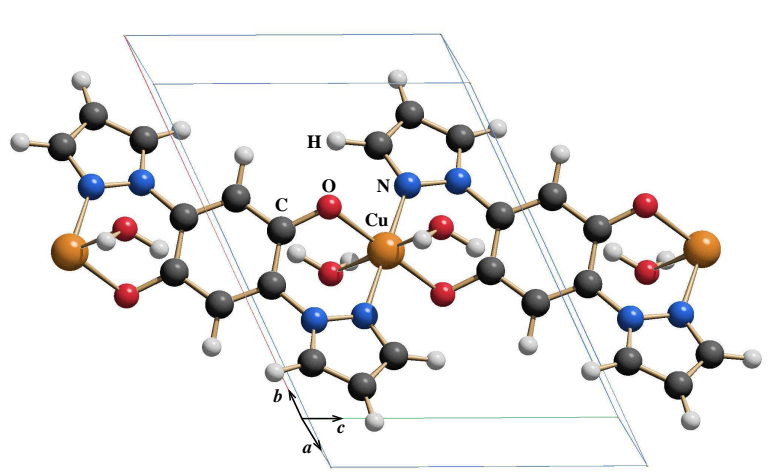

Figure 4: One unit cell of the $[\mathrm{Cu}(\text { bpydhb })]_{n}$ polymer with water molecules bonding to the $\mathrm{Cu}(\mathrm{II})$ ion. The unit cell has been relaxed by the force field method but kept fixed in the AIMD relaxation. It differs considerably from the original $[\mathrm{Cu} \text { (bpydhb) }]_{n}$ polymer unit cell. The equilibrium positions of the water molecule lead to a nearly octahedrally coordinated $\mathrm{Cu}(\mathrm{II})$ ion.

of states for both modifications is presented. While for both cases $\mathrm{Cu}$ is in an oxidation $2+$ with the $d_{x^{2}-y^{2}}$ orbital half filled, the effect of the octahedral crystal field shows a splitting into $t_{2 g}\left(d_{x y}, d_{x z}, d_{y z}\right)$ for energies below $-1.5 \mathrm{eV}$ and $e_{g}\left(d_{z^{2}}, d_{x^{2}-y^{2}}\right)$ states above $-1.5 \mathrm{eV}$ (see Fig. 5 (b)) while in the planar environment all $d$ states are split and the states contributing to the bonding at the Fermi surface are mainly of $d_{x^{2}-y^{2}}$ character in the local coordinate frame of $\mathrm{Cu}$ which is defined with the local $z$ direction pointing from the $\mathrm{Cu}$ to the $\mathrm{O}$ of the $\mathrm{H}_{2} \mathrm{O}$ molecule and the $y$ direction pointing from the $\mathrm{Cu}$ 


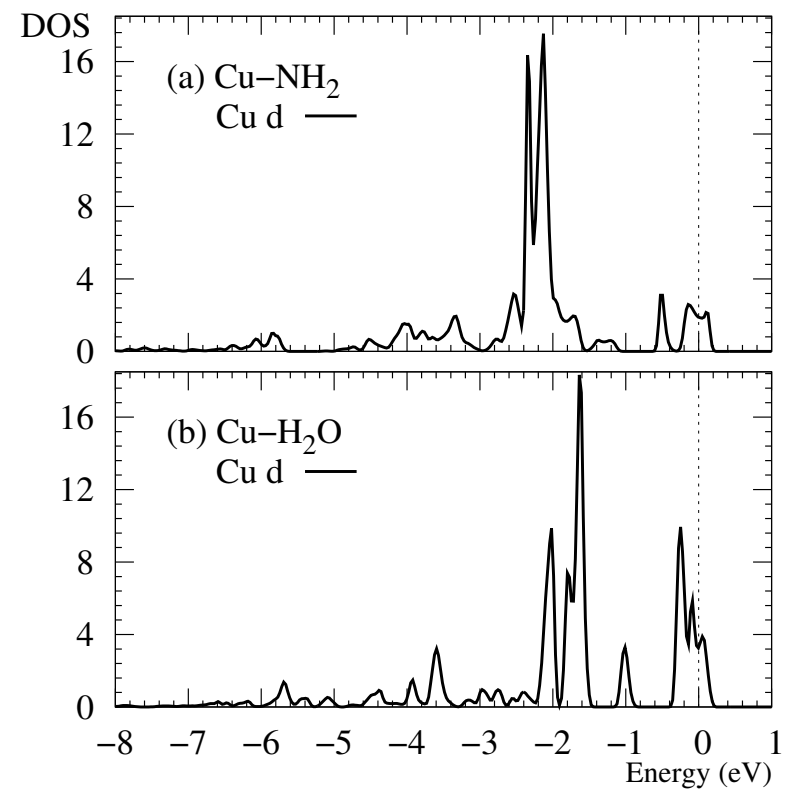

Figure 5: (a) $\mathrm{Cu} d$ density of states of the $\mathrm{Cu}$ polymer $[\mathrm{Cu} \text { (bpydhb) }]_{n}$ with two $\mathrm{NH}_{2}$ groups, and (b) of the $\mathrm{Cu}$ polymer $[\mathrm{Cu}(\text { bpydhb })]_{n}$ with two water ligands.

to the in-plane $\mathrm{O}$ atom.

\section{CONCLUSIONS}

We have investigated the structural properties and its effects on the electronic properties of the quasi-one- dimensional polymer $[\mathrm{Cu}(\mathrm{bpydhb})]_{n}$ with small structural modifications and a model $\mathrm{Fe}$ (II) triazole polymer using classical and $a b$ initio DFT methods. We demonstrate that the use of a stepwise approach to the study of complicated coordination polymer materials is an effective procedure to obtain reliable structures for accurate quantum mechanical analysis. We note that an exclusive use of ab initio molecular dynamics is very time consuming while the combination of the classical with the quantum approaches speeds up the relaxation process considerably. We first employ a classical force field to create and relax plausible initial structures. In a second step, we proceed with $a b$ initio molecular dynamics to relax these structures to a stable configuration. Finally we extract the electronic properties of the transition metal compounds with precise FPLAPW calculations.

\section{Acknowledgments}

H.O.J. gratefully acknowledges support from the DFG through the Emmy Noether Program. This work was financially supported by the Deutsche Forschungsgemeinschaft under the auspices of the Forschergruppe 412 on "spin and charge correlations in low-dimensional metalorganic solids". We thank A. Kokalj for providing the visualization code XcrySDen ${ }^{31}$. We gratefully acknowledge support by the Frankfurt Center for Scientific Computing.
* Electronic address: jeschke@itp.uni-frankfurt.de

1 T. Tayagaki and K. Tanaka, Phys. Rev. Lett. 86 (2001) 2886 (2001).

2 Y. Ogawa, S. Koshihara, K. Koshino, T. Ogawa, C. Urano, and H. Takagi, Phys. Rev. Lett. 84 (2000) 3181.

3 S. Koshihara, Y. Tokura, K. Takeda, and T. Koda, Phys. Rev. Lett. 68 (1992) 1148.

${ }^{4}$ K. Miyano, T. Tanaka, Y. Tomioka, and Y. Tokura, Phys. Rev. Lett. 78 (1997) 4257.

5 See e.g. P. Verwer, F. J. J. Leusen, Rev. Computat. Chem. 12 (1998) 327; W.T.M. Mooij, B.P. van Eijck, S.L. Price, P. Verwer, J. Kroon, J. Computat. Chem. 19 (1998) 459; J.P.M. Lommerse, W.D.S. Motherwell, H.L. Ammon, J.D. Dunitz, A. Gavezzotti, D.W.M. Hofmann, F.J.J. Leusen, W.T.M. Mooij, S.L. Price, B. Schweizer, M.U. Schmidt, B.P. van Eijck, P. Verwer, D.E. Williams, Acta Cryst. B 56 (2000) 697; W. D. S. Motherwell, H. L. Ammon, J. D. Dunitz, A. Dzyabchenko, P. Erk, A. Gavezzotti, D. W. M. Hofmann, F. J. J. Leusen, J. P. M. Lommerse, W. T. M. Mooij, S. L. Price, H. Scheraga, B. Schweizer, M. U. Schmidt, B. P. van Eijck, P. Verwer, D. E. Williams, Acta Cryst. B 58 (2002) 647; G. M. Day, W. D. S. Motherwell, H. L. Ammon, S. X. M. Boerrigter, R. G. Della Valle, E. Venuti, A. Dzyabchenko, J. D. Dunitz, B. Schweizer, B. P. van Eijck, P. Erk, J. C. Facelli, V. E. Bazterra, M. B. Ferraro, D. W. M. Hofmann, F. J. J. Leusen, C. Liang, C. C. Pantelides, P. G. Karamertzanis, S. L. Price, T. C. Lewis, H. Nowell, A. Torrisi, H. A. Scheraga, Y. A. Arnautova, M. U. Schmidt, P. Verwer, Acta Cryst. B 61 (2005) 511.

${ }^{6}$ M.U. Schmidt, U. Englert, J. Chem. Soc., Dalton Trans. 1996 (1996) 2077.

7 J.C. Schön, M. Jansen, Z. Krist. 216 (2001) 307; J.C. Schön, M. Jansen, Z. Krist. 216 (2001) 361.

8 B.P. van Eijck, W.T.M. Mooij, J. Kroon, J. Computat. Chem. 22 (2001) 805.

9 W.T.M. Mooij, F.B. van Duijnefeldt, J.G.C.M. van Duijnefeldt - van de Rijdt, B.P. van Eijck, J. Phys. Chem. A 103 (1999) 9872; W.T.M. Mooij, B.P. van Eijck, J. Kroon, J. Phys. Chem. A 103 (1999) 9883.

10 O. Kahn and C. Jay Martinez, Science, 279 (1998) 44.

11 Y. Garcia, V. Ksenofontov and P. Gütlich, Hyperfine Interactions 139/140 (2002) 543.

12 R. Dinnebier, H. W. Lerner, L. Ding, K. Shankland, W. I. F. David, P. W. Stephens, and M. Wagner, Z. Anorg. Allg. Chem. 628 (2002) 310.

13 B. Wolf, S. Zherlitsyn, B. Lüthi, N. Harrison, U. Löw, V. Pashchenko, M. Lang, G. Margraf, H.-W. Lerner, E. Dahlmann, F. Ritter, W. Assmus, and M. Wagner, Phys. 
Rev. B 69 (2004) 092403.

14 see A. Michalowicz, J. Moscovici, and O. Kahn, J. Phys. IV 7 (1997) 633 and references therein.

15 K. Drabent and Z. Ciunik, Chem. Comm. 14 (2001) 1254.

16 Y. Garcia, P.J. van Koningsbruggen, G. Bravic, P. Guionneau, D. Chasseau, G. L. Cascarano, J. Moscovici, K. Lambert A. Michalowicz, O. Kahn, Inorg. Chem. 36 (1997) 6357.

17 Y. Garcia, P. Guionneau, G. Bravic, D. Chasseau, J.A.K. Howard, O. Kahn, V. Ksenofontov, S. Reiman, P. Gütlich, Eur. J. Inorg. Chem. 2000 (2000) 1531.

18 E. Smit, B. Manoun, S.M.C. Verryn, D. de Waal, Powder Diffraction 16 (2001) 37.

19 see P. Gütlich, Y. Garcia, and H. A. Goodwin, Chemical Physical Society Reviews 29 (2000) 419 and references therein.

${ }^{20}$ G. Vos, R. A. le Febre, R. A. G. de Graaff, J. G. Haasnoot, and J. Reedijk, J. Am. Chem. Soc. 105 (1983) 1682.

${ }^{21}$ Cambridge Structural Database, Cambridge Crystallographic Data Centre, Cambridge, England, 2005.

22 Cerius $^{2}$, Version 4.9. Accelrys Inc., Cambridge/England.

${ }^{23}$ L. Mayo, B.D. Olafson, W.A. Goddard III, J. Phys. Chem.
94 (1990) 8897.

24 J. Gasteiger, M. Marsili, Tetrahedron 36 (1980) 3219.

${ }^{25}$ R. Car and M. Parrinello, Phys. Rev. Lett. 55 (1985) 2471.

26 P. Blaha, K. Schwartz, G. K. H. Madsen, D. Kvasnicka and J. Luitz; WIEN2K, An Augmented Plane Wave + Local Orbitals Program for calculating crystal properties (K. Schwarz, Techn. University Wien, Austria, 2001), ISBN 3-9501031-1-2.

27 P. E. Blöchl, Phys. Rev. B 50 (1994) 17953.

28 J. P. Perdew, K. Burke and M. Ernzerhof, Phys. Rev. Lett. 77 (1996) 3865.

29 L.A. Salguero, H.O. Jeschke, R. Valentí, B. Rahaman, T. Saha-Dasgupta, C. Buchsbaum, M.U. Schmidt, Microscopic ab initio study of the electronic and magnetic properties of triazole-bridged $\mathrm{Fe}(\mathrm{II})$ polymers, in preparation.

30 L.A. Salguero, H.O. Jeschke, R. Valentí, B. Rahaman, T. Saha-Dasgupta, C. Buchsbaum, M.U. Schmidt, cond-mat/0602633.

31 A. Kokalj, Comp. Mater. Sci. 28 (2003) 155. Code available from http://www.xcrysden.org/ 